\title{
Is Resection of Crohn's Disease a Procedure of the Past?
}

\author{
Fabrizio Michelassi
}

Published online: 21 September 2010

(C) Société Internationale de Chirurgie 2010

The first strictureplasty in the treatment of Crohn's disease is attributed to Emanoel Lee in 1976. Lee had become aware of the work by Katariya [1], who performed strictureplasties in tubercular strictures of the terminal ileum. Lee and Papaioannou published their initial experience with strictureplasties in Crohn's disease in 1982 [2]. Lee waited to accrue enough experience because he was fearful that a strictureplasty on chronically diseased bowel would lead to an increased incidence of suture line dehiscences; in addition, he was concerned that leaving gross Crohn's disease behind at the time of the strictureplasty would pave the way for a high symptomatic recurrence rate. Yet his initial experience did not validate these two concerns, and the era of bowel-sparing procedures in Crohn's disease was born.

Strictureplasty techniques were initially advocated for short strictures in the jejunum and ileum. With increasing experience and confidence in these techniques, strictureplasty techniques were adopted to treat strictures at other gastrointestinal sites as well, including the duodenum, the terminal ileum, and even the colon. Poggioli et al. [3] were the first to propose a strictureplasty for terminal ileum disease: they performed a side-to-side anastomosis between the diseased terminal ileum and the ascending colon, which closely mimicked a Finney strictureplasty. A year later Poggioli et al. adopted the side-to-side isoperistaltic strictureplasty devised by Michelassi [4] to neo-terminal ileal disease [5].

F. Michelassi ( $\square)$

Department of Surgery, Weill Cornell Medical College,

New York Presbyterian Hospital-WCMC, 1300 York Avenue,

New York, NY 10065, USA

e-mail: fam2006@med.cornell.edu
Quiescence of disease at the site of a strictureplasty is an observation that surgeons have made over the past two decades when reoperating on patients where a strictureplasty had been performed at a prior procedure. Poggioli et al. [3] were the first to demonstrate and observe quiescence of disease upon endoscopic examination. Histopathologic confirmation of quiescence of disease after a bowel-sparing procedure was provided in a long-term study of side-to-side strictureplasty [6].

The terminal ileum is the gastrointestinal site most often affected by Crohn's disease. Two observations are driving surgeons to devise bowel-sparing procedures for terminal ileal disease: the opportunity to extend bowel-sparing procedures to this site and the observation that, after a strictureplasty, Crohn's disease activity decreases at the operative site.

This article (WJS 10-03-0289.R1) presents the experience the authors have gained with a modification of the side-to-side strictureplasty performed by Poggioli and colleagues. The authors suggest a Finney strictureplasty between the terminal ileum and the right colon where the apex of the strictureplasty is located at the most dependent portion of the diseased terminal ileum, rather than at the ileocecal valve, as in the technique of Poggioli et al. This variation has the intrinsic advantage of accommodating longer segments of diseased terminal ileum than the original technique of Poggioli and colleagues.

The value of this article is that the authors have conducted a feasibility study of a new strictureplasty technique for terminal ileitis. In doing so, they contribute to the concept of bowel-sparing procedures and they do so for an intestinal segment where resection is the standard of care. One disadvantage of the present study is that it comprises a disparate and inhomogeneous group of patients (some with perforating and stenosing disease, some with single-site 
and multiple-site disease, smokers and non-smokers). With this wide inclusion criterion, it is not surprising that the results show that strictureplasty does not confer any protective advantage over resection in terms of local recurrence, a concept that is in disagreement with current data and evidence.

Many questions remain unanswered regarding the use of bowel-sparing surgery for terminal ileal disease. How widely applicable are strictureplasty techniques in terminal ileal disease? The authors performed only 14 ileocecal strictureplasties over a four-year span. Is this procedure applicable only to the rare patient with limited stenosing disease? Will strictureplasty involving the colon and its flora behave differently from strictureplasty involving only the small bowel? Should these patients receive prophylaxis in the form of antibiotics, immunological treatments, or biological therapy? The authors mention that their patients were discharged on mesalamine. Will disease regress endoscopically and pathologically?

It is our hope that this article will stimulate other investigators to carry out prospective and randomized studies to learn about the applicability of this technique in terminal ileitis and its real influence on recurrence rates.

\section{References}

1. Katariya RN, Sood S, Rao PG et al (1977) Strictureplasty for tubercular strictures of the gastrointestinal tract. $\mathrm{Br} \mathrm{J}$ Surg 64:496-498

2. Lee EC, Papaioannou N (1982) Minimal surgery for chronic obstruction in patients with extensive or universal Crohn's disease. Ann R Coll Surg 64:229-233

3. Poggioli G, Stocchi L, Laureti S et al (1997) Conservative surgical management of terminal ileitis: side-to-side enterocolic anastomosis. Dis Colon Rectum 40:234-239

4. Michelassi F (1996) Side-to-side isoperistaltic strictureplasty for multiple Crohn's strictures. Dis Colon Rectum 39:334-345

5. Poggioli G, Selleri S, Stocchi L et al (1998) Conservative surgical management of perforating Crohn's disease: side-to-side neoileocolic anastomosis. Dis Colon Rectum 41:1571-1580

6. Michelassi F, Hurst RD, Melis M et al (2000) Side-to side isoperilstatic strictureplasty in extensive Crohn's disease: a prospective longitudinal study. Ann Surg 232:401-408 